\title{
Improved Quench Localization and Quench Propagation Velocity Measurements in the LHC Superconducting Dipole Magnets
}

\author{
M. Calvi, E. Floch, S. Kouzue, and A. Siemko
}

\begin{abstract}
The series tests performed on the LHC superconducting magnets at cryogenic condition give a unique opportunity to understand the mechanisms responsible for the instabilities causing the so-called training quenches. The first series production tests demonstrated that the weak points in the LHC dipoles are the coil extremities in which the majority of the quenches are located. This evidence triggered the idea of improving the resolution of the measuring system to better characterize the starting region of a quench and the beginning of its propagation. The new quench antennas equipped with sets of small, $4 \mathrm{~cm}$ long, and sensitive pick-up coils were designed. The system is described in detail and the first measurement results are presented. It turned out that this tool is also a reliable technique for measuring the quench propagation velocity especially in the particular regions of the superconducting coils like the crossing between the straight section of the magnet and its extremity.
\end{abstract}

\section{INTRODUCTION}

$\mathbf{T}$ HE Large Hadron Collider (LHC) [1], presently under construction at CERN requires 1232 twin aperture superconducting dipoles magnets, featuring a nominal field of $8.33 \mathrm{~T}$ inside the coil aperture of $56 \mathrm{~mm}$ diameter and a magnetic length of $14.3 \mathrm{~m}$. The cold tests performed on the pre-series LHC dipoles confirmed that the coil extremities are the weak regions where most of the quenches begin. The measurements performed up to now by means of voltage tap technique and the standard low resolution quench antenna [2] give information about which pole of the dipoles is quenching and its longitudinal location within a precision of about half a meter. A careful analysis of the voltage-tap signals indicates which pole layer (inner or outer) is quenching [3]. But the efforts paid to identify the quenching block of cables did not give unique solutions and moreover it was not possible to measure the quench propagation velocity but in few cases. These limitations called for an improvement of the axial resolution to identify the weak elements of the magnet ends. To optimize the resources and to profit from the existing acquisition system, the local

Manuscript received October 3, 2004.

M. Calvi is with the European Laboratory for Nuclear Research, CERN, Geneva CH-1211, Switzerland. He is also with the Geneva University, "Departement de Physique de la Matiere Condensee," Geneva CH-1211, Switzerland (e-mail: marco.calvi@cern.ch).

E. Floch and A. Siemko are with the European Laboratory for Nuclear Research, CERN, Geneva CH-1211, Switzerland (e-mail: eric.floch@cern.ch; andrzej.siemko@cern.ch).

S. Kouzue is with the European Laboratory for Nuclear Research, CERN, Geneva CH-1211, Switzerland. He is also with the J. Fourier University, Grenoble (e-mail: serge.kouzue@ cern.ch).

Digital Object Identifier 10.1109/TASC.2005.849533

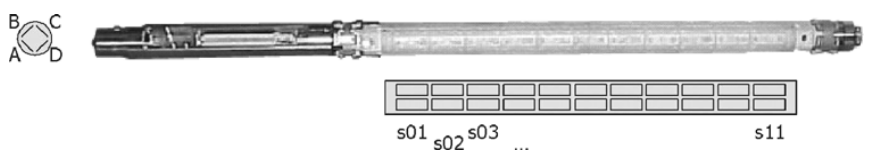

Fig. 1. The final assembly and the cross section of the local quench antenna The pick-up coils are covered with a semi-transparent plastic tube to prevent damages during manipulation.

quench antenna (LQA) has been designed to cover only the problematic region of the magnet. This configuration has the disadvantage that in the rare case in which the quench does not start in the magnet extremities there is no information about its location except that the quench started far from the antenna region.

\section{A. Measurement Principle}

The principles of this measurement technique are explained in detail in [4], [5]. While a superconducting cable is quenching, the current carried in the filaments is shared with the normal conducting stabilizer. In the case of the LHC Rutherford cable it is a copper stabilizer. Because the magnetic field in the cable cross-section is not homogenous and the resistivity of copper increases with the applied magnetic field (magneto resistance) the current redistributes to minimize the dissipated power. This phenomenon is at the origin of a transversal current which generates a temporary local magnetic field distortion. This signal induces a voltage across a pick-up coil. To follow the propagation of the normal conducting front several pick-up coils next to each other are used.

\section{Local Quench ANTENNA Design}

The LQA has been designed to cover the last half meter of the LHC dipole coil. This configuration guarantees to detect quenches which start in the bending region of the coil up to the end of the layer jump region for the connection side. The measuring system operates at room temperature inside an anticryostat [6], although it is possible to adapt it for operating in cold conditions.

The pick-up coils assembled in the LQA are wound on glassreinforced epoxy which is $4 \mathrm{~cm}$ long, $1 \mathrm{~cm}$ wide and $1 \mathrm{~mm}$ thick. On each pick-up coil, the signal is carried out via a twisted wire-pair soldered on the upper face of the coil, inside of a small connector. The shaft on which the pick-up coils are assembled is composed of eleven longitudinal sections. Each of them holds four pick-up coils in tangential configuration (see Fig. 1). Four grooves, beside each longitudinal array of pick-up coils, 


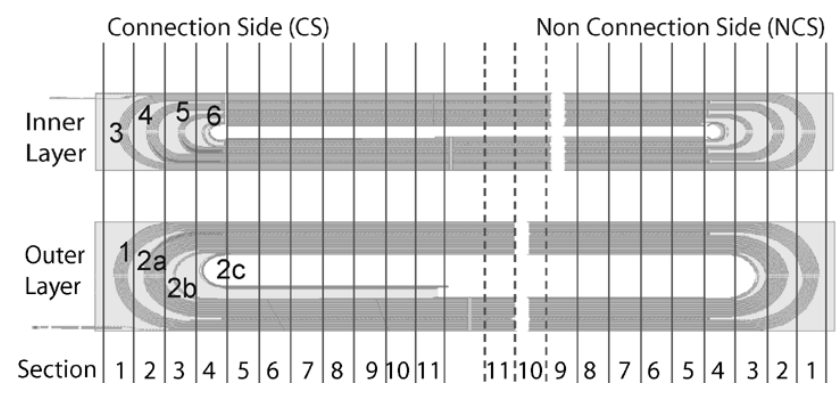

Fig. 2. The standard longitudinal position of the LQA with respect to the LHC dipole superconducting coils. The sections s01, s02, s03, s04 are dedicated to the monitoring of the bending region of the magnet. The label on each cable block is used as a reference to identify the quenching region.

are used to place the twisted wires. At the end of each groove the wires enter a hole and arrive at two rectangular connectors fixed in a titanium support. This unit has in its extremity a mechanical connection to join with extension modules about $1.5 \mathrm{~m}$ long equipped with two multifilament cables that are plugged on the antenna connectors to carry the signals to the acquisition system. Thanks to the modular system adopted, the antenna can be placed all along the magnet by a single person. A mechanical support has been designed to help the installation and to give a precise longitudinal and azimuthal position to the antennas.

\section{A. Experimental Set-Up}

The standard operating configuration foresees that four antennas are installed, one in each magnet extremity. The longitudinal position of two LQA's, with respect to the LHC dipole magnet coil longitudinal section, is illustrated in Fig. 2. The winding structure in the extremities is more complex than in the straight part of the magnet. The 2nd block splits in two in the nonconnection side and in three coils in the connection side. In the following, the sub-parts of the second block are called a, b, c, starting from the outside (Fig. 2). Each antenna is fixed with the coils at $45^{\circ}$ degree with respect to the main field. The pick-up coil signal is affected by the main field variation. To eliminate this signal component the difference between two pick-up coils with the same polarization with respect to the main field is taken before entering the acquisition system. This is also a fair method to reduce the number of signals. If the pairs of pick-up coils used for the compensation are far enough each from each other, the signal induced by the quench has an amplitude very different on both. The compensation schema adopted is the following

$$
\left\{\begin{array}{l}
V_{A C}=V_{A}+V_{C}, \\
V_{B D}=V_{B}+V_{D},
\end{array}\right.
$$

where $V_{A}, V_{C}, V_{B}, V_{D}$ are the raw voltage of the coils with their own polarization (Fig. 1). The convention chosen for the polarization is the one which preserves the rotation invariance of the coils with the positive versus going out the shaft.

\section{DATA ANAlysis PROCEDURE}

Several LHC dipole magnets have been tested with the LQA. The tests results have shown that the antennas are sensitive and selective enough to discriminate the starting region of the
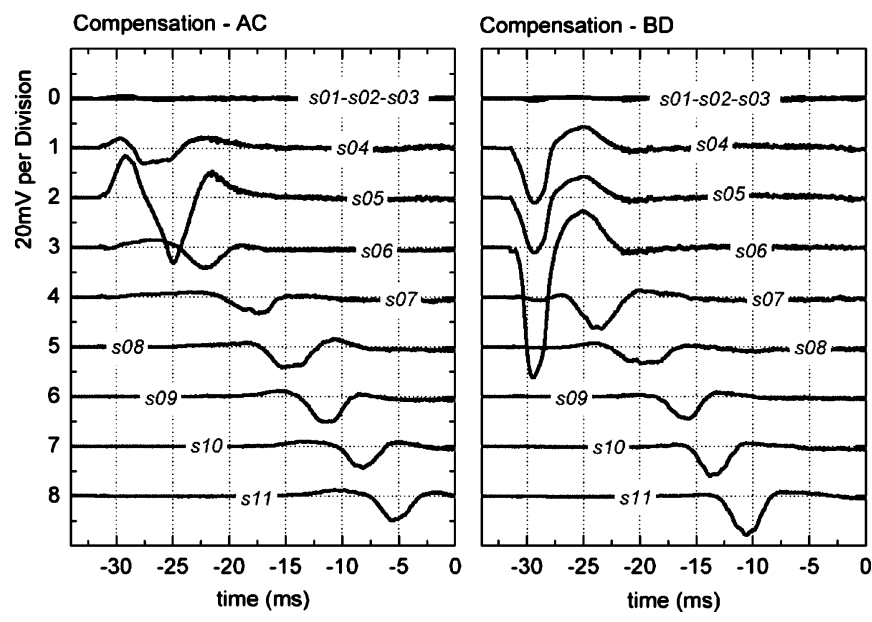

Fig. 3. The signals recorded on the antenna located in the quenching extremity. Different sections are spaced in amplitude of $20 \mathrm{mV}$ to easy inspection. The sections s01-s02-s03 are not activated and they are plotted on the same raw. The signals are filtered to reject the high frequency noise.

quench among different blocks and accurately measure the quench propagation velocity. Clear examples of sensitivity to left-right position of the quench have been observed in agreement with predictions. In the following subsections, an example of data is discussed and the procedures used for the analysis are described.

\section{A. Raw Data Examples}

An example of raw data acquisition is presented in Fig. 3. Before the beginning of the quench the signal across the pick-up coils is at zero average, which indicates that the compensation technique efficiently eliminates the induced voltage due to the current ramping $(10 \mathrm{~A} / \mathrm{s})$ and the power supply ripples. When the quench starts the sections s04, s05, s06 are activated at the same time. Signals are present on both AC and BD compensation (with opposite sign) but are higher on BD. The highest signal is on s06-BD. After a transitory regime of about $5 \mathrm{~ms}$, in which the signals oscillate, coil s07-BD displays a major single bump-shape. With an almost constant delay the signals appear consequently in s08-09-10-11 consistent with steady normal front propagation. With AC compensations the same phenomenon is visible, but each signal is delayed about $5 \mathrm{~ms}$ with respect to the same section in the BD compensation. The AC signals show the propagation of the second front. It is evident that the two front signals are not fully decoupled. For instance, the signal s11-BD has a negative minimum at $-10 \mathrm{~ms}$ which corresponds to a positive maximum 8 times attenuated in the s11-AC.

\section{B. Quench Localization}

Localizing the starting region of the quench is very important to understand the performance of a superconducting magnet. The first pick-up coil activated in the antenna gives the longitudinal location of the transition region. If more than one coil is activated at the same time, the whole region covered by the activated coils is considered quenching. If the readout signal of one coil is significantly higher than the others, the hot spot region 


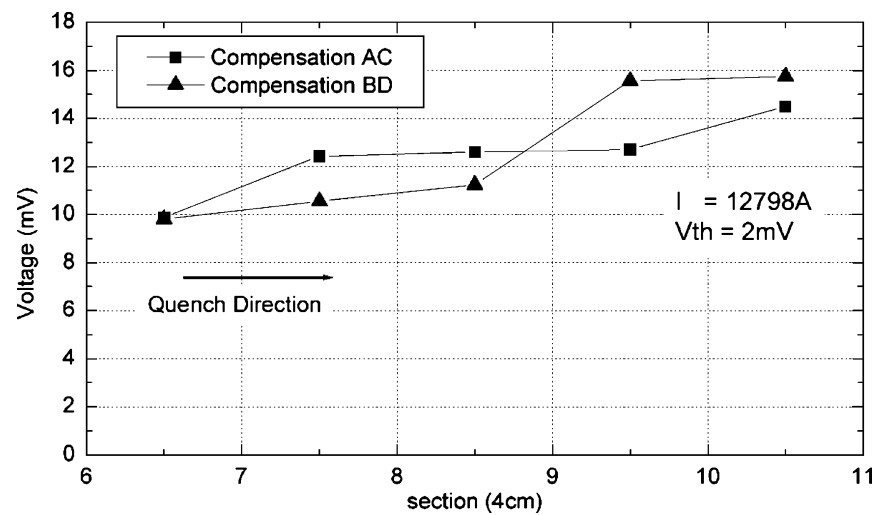

Fig. 4. The average quench propagation velocity between two neighboring sections of the LQA. The measurements presented in this example are extracted from the same raw data presented previously. The analysis has been performed using a threshold value of $2 \mathrm{mV}$.

of the starting quench is considered to be closer to that coil. If two coils are activated at the same time, with about the same intensity, the quench hot-spot is considered located in the middle. The magnet cross section is divided in blocks (Fig. 2) and this structure is preserved in the extremities. This geometry helps to identify the quenching block because each of them bends at different longitudinal position and each of them is spaced by about one pick-up coil length, as shown in Fig. 2. Wherever the quench starts in the extremity, it propagates and reaches the bending part. The last coil activated during the propagation toward the extremity reveals the quenching block. For instance, in the raw data of Fig. 3, the last activated pick-up coils are in s04 which, knowing that the quench was in the inner layer pole, lets conclude that the quenching block was the 6 th.

\section{Quench Propagation Velocity}

The quench propagation velocity is a very important parameters for quench protection studies, because it gives the major contribution to the voltage-drop development across the magnet. Unfortunately, it is not easy to estimate with good precision the quench propagation velocity of a cable even with sophisticated numerical simulation. The quench antenna is a fairly simple instrument to measure it. The easiest criterion to extract the information about velocity is to define a threshold above which the quench is considered to be at the beginning of a coil. The time taken to observe the signal in the next coil at the same voltage level is considered equivalent to the time the quench takes to go from a coil to the next $(\delta t)$. Knowing the distance between the beginning of two pick-up coils $(l)$, the velocity is simply $v_{q}=l / \delta t$. Because the antenna is equipped with many sections of pick-up coils, the velocity can be measured as a function of time or space (Fig. 4). During 5 to $10 \mathrm{~ms}$ after the quench start, it is difficult to measure the quench propagation velocity because the front is not yet established. This transition period is related to the conditions in which the quench is initiated. In some cases, the transition period gives higher velocity than the stationary state. In other cases, transition velocities are lower. The signal associated to the steady quench propagation velocity does not depend on the starting conditions, which change from quench to quench and it has a simpler interpretation.

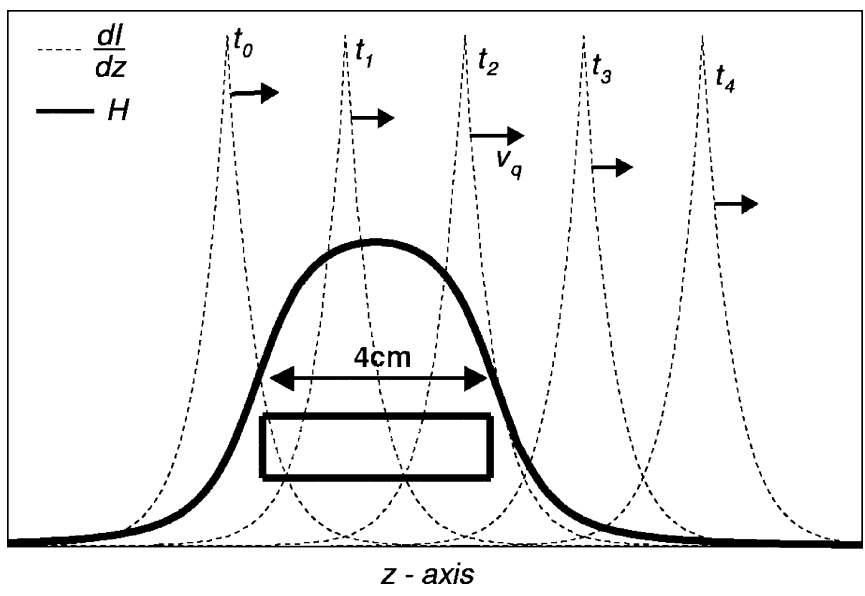

Fig. 5. The schematic view of the coupling mechanism between the quench front and a pick-up coil. The current distribution moves with the quench front at constant velocity $v_{q}$ while the pick-up coil is fixed. The voltage across the pick-up coil is proportional to the value of the space integral between $d I / d z$ and $H$.

\section{The Quench Propagation Signal}

A few milliseconds after the quench start, the pick-up coil signals become very similar to each other. This is the experimental evidence that the quench propagation front, causing the current redistribution, becomes stable in the axial direction and is moving at constant velocity $v_{q}$. In the following, a model of two superconducting wires is introduced to interpret the experimental data, while details of the calculations can be found in [7]. The voltage across the pick-up coil, as a function of the current distribution can be formulated:

$$
v(t)=\frac{d}{d t} \int_{-\infty}^{+\infty}\left[I_{h f}(z, t) h_{h f}(z)+I_{l f}(z, t) h_{l f}(z)\right] d z
$$

where $I_{h f}$ and $I_{l f}$ are the currents along the wire located in the high field region and in the low field region respectively, $h_{h f}$ and $h_{l f}$ are the transfer functions of the pick-up coils, they are different because the two wires have different location. If the quench front is translation invariant at the velocity of the quench $\left(v_{q}\right)$ then (2) can be simplified as

$$
v(t)=-v_{q} \int_{-\infty}^{+\infty} \frac{d I}{d z}\left(z-v_{q} t\right) H(z) d z,
$$

where

$$
H(z)=h_{h f}(z)-h_{l f}(z)
$$

Equation (3) is essentially a convolution product. The coupling mechanism is sketched in Fig. 5. The current redistributes smoothly from the high field side to the low field side whose resistance is lower because the field is lower. The current derivative with respect to space $(d I / d z)$ and the transfer function $(H)$ are both single bump functions. Their convolution is also a single bump, which fits well the experimental pick-up voltage. Moreover knowing the location of the quenching cable and the associated transfer function, the current distribution in the stationary regime can be estimated directly from measurements by inverting (3). Sometimes the first bump is directly followed 
by a second one, which has opposite sign and much lower amplitude. This latter can be interpreted as the voltage induced by the current coming back to the high field region This is related essentially to the increasing resistivity above $20 \mathrm{~K}$. The fraction of current redistributed between the high field and low field region is

$$
\Delta I_{0}=\frac{R_{h f}-R_{l f}}{R_{h f}+R_{l f}} I_{t o t},
$$

where the $R_{h f}$ and $R_{l f}$ are the linear resistivity of the high and the low field region respectively. When the temperature goes above $20 \mathrm{~K}$ the sum of $R_{h f}$ and $R_{l f}$ increases, with the effect of decreasing $\Delta I_{0}$.

\section{A. The Transfer Function of the Pick-Up Coil}

In the evaluation of the pick-up coil transfer function, several models have been considered. The segment-like approximation has been chosen to discuss the results because it has a simple analytical expression, which it is accurate enough. It can be formalized as follow:

$$
\begin{aligned}
h(z) & =\frac{\mu_{0} N_{t} w}{4 \pi d^{2}} \hat{t} \cdot \hat{n}(\tilde{h}(z+L)-\tilde{h}(z)), \\
\tilde{h}(z) & =\frac{z}{\sqrt{z^{2}+d^{2}}}, \\
\hat{t} & =\frac{\hat{z} \times \hat{r}}{|\hat{z} \times \hat{r}|},
\end{aligned}
$$

where $N_{t}$ is the number of turns, $L$ the length and $w$ the width of the pick-up coil. $d$ is the distance between the center of the antenna and wire, $\hat{n}$ is the unit vector normal to the pick-up mid-plane surface and $\hat{t}$ is the unit vector normal to the plane, which contains the wire and the center of the pick-up coil.

\section{B. Sensitivity to Left-Right Position of the Quench}

If the current redistribution zone is shorter than the pick-up coil length then (3) gets simpler

$$
V(t)=-v_{q} \Delta I_{0} H\left(-v_{q} \cdot t\right),
$$

and the ratio between the maximum values of the two compensation can be evaluated. This simple calculation is in fair agreement with the more accurate results found in [7]. Considering the LHC cross section, it is always true that the compensation which has the highest signal is the one which has one pick-up coil in the quadrant where the quench starts.

\section{Summary of the First Test CAmpaign}

During the first test campaign, the measurements performed with the LQA helped in understanding the origin of low quench performance in several problematic magnets. The training quenches detected in the magnet extremities started in the sixth and in the second block. The quenches located in the 2 nd block started only in the sub-parts b and c. The longitudinal position of those quenches was located in a region of few centimeters close to the magnet extremities, in some cases in the bending part where the cable begin to turn and in other cases about $4 \mathrm{~cm}$
TABLE I

SUMMARY OF THE SYSTEMATIC QUENCH LOCATIONS

\begin{tabular}{lr}
\hline \hline Quench Location & $\%$ \\
\hline $2^{r d}$ b Block (CS/NCS) & 17.6 \\
$2^{t h} \mathrm{c}$ Block (CS) & 20.6 \\
$6^{\text {th }}$ Block (CS/NCS) & 38.3 \\
Straight Part & 23.5 \\
\hline
\end{tabular}

before. Three pick-up coils are usually activated at the same time in the very beginning of the resistive transition which suggests that the starting quench has a dimension of maximum $12 \mathrm{~cm}$, comparable to the transposition pitch length. This last experimental evidence supports the hypothesis that the quench nucleation is generated by mechanical instabilities in between the two layers of strands in the Rutherford cable (Table I).

\section{CONCLUSIONS}

The local quench antenna was demonstrated to be a fair instrument to identify the quenching block of cables in the LHC dipole magnets with a resolution of few centimeters and with a strong sensitivity to left-right position. Moreover it has provided precise measurements of the quench propagation velocity as a function of time and space, which help to understand the voltage drop across the magnet during a quench. The LQA improved the feed back to the magnet production, and it provided useful information to the magnet technology community.

\section{ACKNOWLEDGMENT}

The authors would like to acknowledge F. Thierry and M. Genet for their contribution during the design effort, F. Boisier and P. Legrand for their help during the assembly, G. D'Angelo and M. Gateau for the integration of the system within the existing electronic and V. Chohan and the test operation team for their help during the measurements. The authors are also grateful to Prof. R. Flükiger for his constant support during this project.

\section{REFERENCES}

[1] “The LHC Main Ring,” LHC Design Report, CERN-2004-003, vol. 1, Jun. 2004.

[2] J. Billan et al., "Twin rotating coils for cold magnetic measurements of $15 \mathrm{~m}$ long LHC dipoles," IEEE Trans. Appl. Supercond., vol. 10, no. 1, pp. 1422-1426, Mar. 2000.

[3] E. Floch, private communication to be published as a CERN project note.

[4] D. Leroy, J. Krzywinski, V. Remondino, L. Walckiers, and R. Wolf, "Quench observation in the LHC superconducting one meter long models by field perturbation measurements," in Proc. Applied Superconducting Conf., Chicago, Aug. 23-28, 1992.

[5] A. Siemko, J. Billan, G. Gerin, D. Leroy, L. Walckiers, and R. Wolf, "Quench localization in the superconducting model magnets for the LHC by means of pick-up coils," IEEE Trans. Appl. Supercond., vol. 5, pp. 1028-1031, Jun. 1995.

[6] O. Dunkel, P. Legrand, and P. Sievers, "A warm bore anticryostat for series magnetic measurements of LHC superconducting dipole and short straight section magnets," presented at the CEC/ICMC 2003, Anchorage, Alaska, Sep. 22-26, 2003.

[7] A. Forrester and M. Calvi, "Modeling quench signals in the LHC superconducting dipoles," IEEE Trans. Appl. Supercond., submitted for publication. 